\title{
Comparative study of a constant-alpha force-free field and its approximations in an ideal toroid
}

\author{
M. Vandas ${ }^{1}$ and E. Romashets ${ }^{2}$ \\ 1 Astronomical Institute, Academy of Sciences of the Czech Republic, Boční II 1401, 14100 Praha 4, Czech Republic \\ e-mail: vandas@asu.cas.cz \\ 2 Lone Star College, Houston, TX77088 Texas, USA \\ e-mail: romash7@gmail.com
}

Received 2 April 2015 / Accepted 19 June 2015

\begin{abstract}
Aims. Magnetic clouds in the solar wind are large, loop-like interplanetary flux ropes and may be locally approximated by a toroidal flux rope. We compare approximate constant-alpha force-free fields in an ideal toroid, used in magnetic cloud analysis, with the exact solution, and examine their validity for low aspect ratios, which can be found in magnetic clouds. The approximate toroidal solutions were originally derived under the assumption of large aspect ratios.

Methods. Three analytic simple approximate constant-alpha force-free solutions and the exact analytic solution are compared with respect to magnetic field profiles, magnetic field magnitude distributions, and magnetic helicity, with moderate (2-3) and very low $(<2)$ aspect ratios.

Results. The Miller \& Turner (1981, Phys. Fluids, 24, 363) field and its modification (to satisfy exact solenoidality) match the position of the magnetic axis in the toroidal flux rope well even for very low aspect ratios. The same can be said for the modified field and the position of the magnetic field maximum. When calculating helicity of the toroidal flux rope, the Miller \& Turner field yields better results. A simple formula for magnetic helicity derived from the Miller \& Turner solution is valid with a good accuracy even for very low aspect ratios.

Conclusions. The Miller \& Turner solution is a reasonable substitute for the exact solution even for low aspect ratios $(\approx 2)$.
\end{abstract}

Key words. solar wind - magnetic fields - magnetohydrodynamics (MHD)

\section{Introduction}

It is widely accepted that magnetic flux ropes are quite commonly observed in the solar wind. Their first in-situ observations were reported during the 1970s (Krimigis et al. 1976). Large and relatively cold interplanetary flux ropes were labelled magnetic clouds (Burlaga et al. 1981; Klein \& Burlaga 1982; Burlaga \& Behannon 1982), and their relationship to coronal mass ejections coming from the Sun was found (Burlaga et al. 1982). More detailed examinations revealed that, aside from magnetic clouds, there were many smaller-scale flux ropes present in the solar wind (Shimazu \& Marubashi 2000; Moldwin et al. 2000; Mandrini et al. 2005; Cartwright \& Moldwin 2010). Burlaga (1988) suggested using a simple constant-alpha force-free field in a cylindrical flux rope (derived by Lundquist 1950) to model a magnetic field configuration inside magnetic clouds. This configuration proved to be very useful for the evaluation of magnetic cloud parameters (e.g. axis orientation, radius, helicity) and, within this model, these parameters are nearly routinely estimated for all identified magnetic clouds (cf. Lepping et al. 1990, 2006, 2015), as well as in some small-scale flux-rope studies (e.g. Shimazu \& Marubashi 2000).

It is evident that modelling an interplanetary-flux-rope magnetic configuration by a cylindrical flux rope can only be done locally. Interplanetary flux ropes are bent and, at least for some time, they remain connected by their feet to the Sun (Burlaga et al. 1990; Chen \& Garren 1993; Marubashi 1997; Janvier et al. 2013). In some cases curvature of flux ropes must be taken into account to correctly interpret magnetic field observations inside interplanetary flux ropes (Ivanov et al. 1989; Marubashi 1997; Romashets \& Vandas 2003a; Marubashi \& Lepping 2007; Hidalgo 2014). Usually it is a local fitting because the model is an ideal torus. Hidalgo $(2013,2014)$ recently published a global model of an interplanetary flux rope, which has a varying cross section and a non-circular axis. It is a non-force-free model. In the present paper, we deal with linear force-free configurations. In that case, a flux rope is locally modelled by an ideal toroid either with a toroidally adjusted Lundquist solution (Marubashi 1997), or with the Miller \& Turner (1981) solution (Ivanov et al. 1989). Toroidally adjusted Lundquist solution is a very rough approximation and the Miller and Turner solution was derived under an assumption of large aspect ratios (the ratios between the major, $R_{0}$, and minor, $r_{0}$, radii of a toroid). Geometrical considerations (Marubashi 1997) or magnetohydrodynamic simulations (Vandas et al. 2002) indicate that there are bent parts of interplanetary flux ropes where the curvature is quite high, and hence aspect ratios are relatively low. It raises the question of whether these parts can be meaningfully fitted by the above mentioned solutions, however, Tsuji (1991) proposed the solution of a linear force-free configuration in an ideal toroid of an arbitrary aspect ratio, which is not widely known in the space-research community. In the next section, we describe this solution and list approximate linear force-free toroidal solutions used for interpretation of magnetic cloud observations. The Tsuji solution is computationally expensive and not very easy to implement in comparison with the approximate solutions, which are very simple. Therefore, for practical reasons the approximate solutions would be preferred, and we check here how they compare with 
the exact solution, especially for lower aspect ratios. Some preliminary comparisons have been made in our proceeding papers (Romashets \& Vandas 2003b; Vandas \& Romashets 2010), and here we present more comprehensive comparisons, with special emphasis on magnetic helicity when aspect ratio is moderate or very low. Validity of a simple analytic formula for helicity, derived from the Miller and Turner solution, is examined for low aspect ratios.

\section{Toroidal constant-alpha force-free models}

Linear force-free field $\boldsymbol{B}$ fulfills the condition

$\operatorname{rot} \boldsymbol{B}=\alpha \boldsymbol{B}$,

where $\alpha$ is a constant ( $\alpha \neq 0)$, hence this force-free field is also called a constant-alpha force-free field. The sign of $\alpha$ determines the chirality of the field. Applying div operator to Eq. (1), we see that the field is automatically solenoidal.

The Lundquist (1950) linear force-free field in a cylindrical flux rope, given in cylindrical coordinates $r, \varphi$, and $Z$, reads

$B_{r}^{(\mathrm{L})}=0$,

$B_{\varphi}^{(\mathrm{L})}=B_{0} J_{1}(\alpha r)$

$B_{Z}^{(\mathrm{L})}=B_{0} J_{0}(\alpha r)$,

where $J_{n}$ are the Bessel functions of the first kind, $B_{0}$ scales the magnetic field and it is also the value of the field maximum located at the flux rope axis $(r=0)$. Each $r=$ const. is a magnetic surface, hence, each cylinder may form a flux rope. The component $B_{Z}$ reaches its maximum at the axis and then monotonously decreases to zero at $\alpha r=a_{0}$, where $a_{0}$ is the first root of $J_{0}$ $\left(a_{0} \approx 2.405\right)$. Usually, the flux rope boundary is set at this place, so $\alpha$ is related to the flux rope radius $r_{0}$ by

$\alpha= \pm a_{0} / r_{0}$.

The toroidally adjusted Lundquist solution simply results from an ad-hoc change of field components in cylindrical coordinates into toroidally curved cylindrical coordinates $r, \varphi$, and $\theta$ (Fig. 1a),

$x=\left(R_{0}+r \cos \theta\right) \cos \varphi$,

$y=\left(R_{0}+r \cos \theta\right) \sin \varphi$,

$z=r \sin \theta$,

and it reads

$B_{r}^{(\mathrm{LT})}=0$,

$B_{\varphi}^{(\mathrm{LT})}=B_{0} J_{0}(\alpha r)$

$B_{\theta}^{(\mathrm{LT})}=-B_{0} J_{1}(\alpha r)$.

One cannot expect that this field to be solenoidal or force-free. The boundary of our toroid is determined by $r=r_{0}, 0 \leq \theta \leq 2 \pi$, $0 \leq \varphi \leq 2 \pi$, and its volume by $0 \leq r \leq r_{0}, 0 \leq \theta \leq 2 \pi$, $0 \leq \varphi \leq 2 \pi$.

The Miller \& Turner (1981) solution was derived under assumption of a large aspect ratio $R_{0} / r_{0}$ of a toroid and in its simplest form, used in magnetic cloud analyses, it reads

$B_{r}^{(\mathrm{MT})}=\frac{B_{0}}{2 \alpha R_{0}} J_{0}(\alpha r) \sin \theta$

$B_{\varphi}^{(\mathrm{MT})}=B_{0}\left(1-\frac{r}{2 R_{0}} \cos \theta\right) J_{0}(\alpha r)$,

$B_{\theta}^{(\mathrm{MT})}=-B_{0}\left\{J_{1}(\alpha r)-\frac{1}{2 \alpha R_{0}}\left[J_{0}(\alpha r)+\alpha r J_{1}(\alpha r)\right] \cos \theta\right\}$.
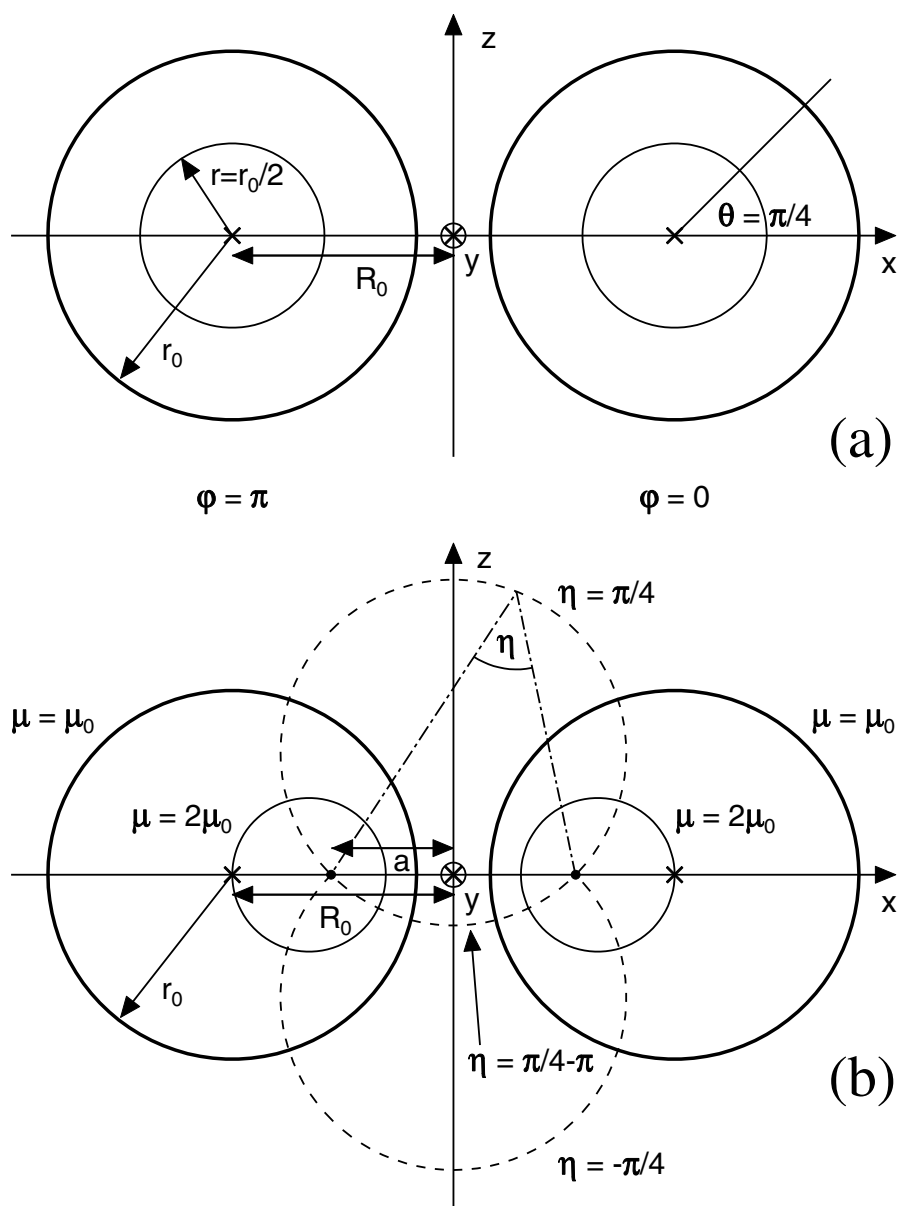

Fig. 1. Two coordinate systems shown separately in a) and b), that we used for descriptions of toroidal magnetic field configurations. Cross sections of our toroid's surface (with major and minor radii, $R_{0}$ and $r_{0}$, respectively) with the $x z$ plane are shown with the thick circles. Our toroid has a circular axis at $x^{2}+y^{2}=R_{0}^{2}$ in the $z=0$ plane; cross sections of this circular axis with the $x z$ plane indicate two symmetric crosses (outside the origin). A common coordinate of the systems is $\varphi$, a usual azimuthal angle in the $x y$ plane; it is $\varphi=0$ for the halfplane $(x>0) z$ and $\varphi=\pi$ for the half-plane $(x<0) z$, as labelled in the figure. a) Toroidally curved cylindrical coordinates have $r$ and $\theta$ coordinates, in addition. The $r$ coordinate defines toroidal surfaces, which have circular axes identical with our toroid; cross sections of one of these surfaces with the $x z$ plane are shown with thin circles; it is $r=r_{0} / 2$. The $\theta$ coordinate is a positional angle. b) Toroidal coordinates have $\mu$ and $\eta$ coordinates, in addition. The $\mu$ coordinate defines toroidal surfaces, which have co-centric, but different circular axes lying in the $z=0$ plane. The circular axes tend to an asymptotic axis, which fulfills $x^{2}+y^{2}=a^{2}$. Its cross sections with the $x z$ plane are shown with two symmetric bullets at the $x$ axis. The surface of our toroid is defined as $\mu=\mu_{0}, \cosh \mu_{0}=R_{0} / r_{0}$. Cross sections of a toroidal surface defined by $\mu=2 \mu_{0}$ with the $x z$ plane are shown with thin solid circles. The coordinate $\eta$ defines spherical surfaces with centers at the $z$ axis. Cross sections of three spherical surfaces $(\eta= \pm \pi / 4, \pi / 4-\pi)$ with the $x z$ plane are plotted as arcs by the dashed line. The absolute value of the coordinate $\eta$ of a point is a viewing angle under which the asymptotic circular axis is seen (it is demonstrated in the figure; the viewing angle is drawn by the dotted-dashed line and its value is $\eta=\pi / 4$ ).

The Miller \& Turner solution is not exactly solenoidal. We tried to fix this problem (Romashets \& Vandas 2003b) and suggested a modified Miller \& Turner solution, derived via the magnetic vector potential. The magnetic vector potential $\boldsymbol{A}$ is defined by

$\boldsymbol{B}=\operatorname{rot} \boldsymbol{A}$, 
and can be easily specified for a linear force-free field by

$\boldsymbol{A}=\boldsymbol{B} / \alpha$

because $\operatorname{rot} \boldsymbol{A}=\operatorname{rot} \boldsymbol{B} / \alpha=\boldsymbol{B}$. Strictly speaking, the magnetic vector potential of the Miller \& Turner solution does not exist because this field is not exactly solenoidal. Hence, the expression $\boldsymbol{B}^{(\mathrm{MT})} / \alpha$ from Eq. (16) is not the magnetic vector potential of the Miller \& Turner solution in an exact sense, but we regard it as a magnetic vector potential of a field, which we call the modified Miller \& Turner (mMT) solution,

$\boldsymbol{A}^{(\mathrm{mMT})}=\boldsymbol{B}^{(\mathrm{MT})} / \alpha$,

so the modified Miller \& Turner solution can be expressed as

$\boldsymbol{B}^{(\mathrm{mMT})}=\operatorname{rot} \boldsymbol{A}^{(\mathrm{mMT})}=\operatorname{rot} \boldsymbol{B}^{(\mathrm{MT})} / \alpha$,

explicitly,

$$
\begin{aligned}
B_{r}^{(\mathrm{mMT})}= & B_{0} \frac{R_{0}-2 r \cos \theta}{2 \alpha R_{0}\left(R_{0}+r \cos \theta\right)} J_{0}(\alpha r) \sin \theta, \\
B_{\varphi}^{(\mathrm{mMT})}= & B_{0}\left(1-\frac{r}{2 R_{0}} \cos \theta\right) J_{0}(\alpha r), \\
B_{\theta}^{(\mathrm{mMT})}= & -\frac{B_{0}}{2 \alpha R_{0}\left(R_{0}+r \cos \theta\right)}\left\{2 \alpha R_{0}^{2} J_{1}(\alpha r)\right. \\
& -R_{0}\left[J_{0}(\alpha r)-\alpha r J_{1}(\alpha r)\right] \cos \theta \\
& \left.+r\left[2 J_{0}(\alpha r)-\alpha r J_{1}(\alpha r)\right] \cos ^{2} \theta\right\} .
\end{aligned}
$$

For large aspect ratios, the Miller \& Turner solution is close to a linear force-free field, so the modified Miller \& Turner solution is close to the Miller \& Turner solution because of the definition (18). In addition, the modified Miller \& Turner solution is exactly solenoidal for any aspect ratio because $\operatorname{div} \boldsymbol{B}^{(\mathrm{mMT})}=$ $\operatorname{div} \operatorname{rot} \boldsymbol{A}^{(\mathrm{mMT})}=0$ (as every field defined by a vector potential).

These three toroidal solutions have $B_{r}=0$ at the toroid's boundary $r=r_{0}$ when $\alpha$ is given by Eq. (5), and so their magnetic field configurations are confined into an ideal toroid (with circular cross section), they are axisymmetric (with respect to the rotational axis $(z)$, i.e. the fields do not depend on $\varphi$ ), their axial fields $\left(B_{\varphi}\right)$ are zero at the boundary, and they tend to the Lundquist solution for large aspect ratios $\left(R_{0} / r_{0} \rightarrow \infty\right)$. In that sense, they represent a toroidal counterpart of the Lundquist solution. However, they are only approximately force-free: with accuracy expressed in $r_{0} / R_{0}$, this condition is fulfilled as $O(1)$ in case of the toroidally adjusted Lundquist solution and as $O\left(r_{0} / R_{0}\right)$ for the Miller \& Turner and modified Miller \& Turner solutions; and the same holds for accuracy of solenoidality for the first two solutions (Romashets \& Vandas 2003b; Vandas \& Romashets 2010). The genuine toroidal counterpart of the Lundquist solution follows from the Tsuji (1991) solution. This solution is formulated for a general cross section and it was not very easy to extract our case from the given paper. We find it useful to present this special case here in a form enabling a straightforward implementation available for magnetic cloud or other research. The solution is exactly solenoidal and force-free for any aspect ratio.

The Tsuji solution is given in toroidal coordinates $\mu, \eta$, and $\varphi$ (Fig. 1b) determined by the following relationships to Cartesian coordinates:

$$
\begin{aligned}
& x=\frac{a \sinh \mu \cos \varphi}{\cosh \mu-\cos \eta}, \\
& y=\frac{a \sinh \mu \sin \varphi}{\cosh \mu-\cos \eta}, \\
& z=\frac{a \sin \eta}{\cosh \mu-\cos \eta} .
\end{aligned}
$$

The parameter $a>0$ scales the system. Each coordinate $\mu=$ const. $>0$ represents a surface of a toroid (while $\mu \geq 0$ in general). When $a=\sqrt{R_{0}^{2}-r_{0}^{2}}$, then our toroid has $\mu=\mu_{0}$, $\cosh \mu_{0}=R_{0} / r_{0}$. The angle $\varphi$ is an azimuthal (rotational) angle of the toroid, $\varphi \in\langle 0,2 \pi)$, and the angle $\eta \in\langle-\pi, \pi\rangle$ complements the coordinates. The boundary of our toroid is determined by $\mu=\mu_{0},-\pi \leq \eta \leq \pi, 0 \leq \varphi \leq 2 \pi$, and its volume by $\mu \geq \mu_{0}$, $-\pi \leq \eta \leq \pi, 0 \leq \varphi \leq 2 \pi$.

The field is

$$
\begin{aligned}
& B_{\mu}^{(\mathrm{T})}=\frac{1}{\alpha h_{\eta} h_{\varphi}} \frac{\partial}{\partial \eta}\left(h_{\varphi} B_{\varphi}\right), \\
& B_{\eta}^{(\mathrm{T})}=-\frac{1}{\alpha h_{\mu} h_{\varphi}} \frac{\partial}{\partial \mu}\left(h_{\varphi} B_{\varphi}\right), \\
& B_{\varphi}^{(\mathrm{T})}=\frac{1}{h_{\varphi}} \sum_{l=0}^{\infty} g_{l} S_{l}(\mu, \eta)
\end{aligned}
$$

with the Lamé coefficients

$h_{\mu}=h_{\eta}=\frac{a}{\cosh \mu-\cos \eta}, \quad h_{\varphi}=\frac{a \sinh \mu}{\cosh \mu-\cos \eta}$,

and

$S_{l}(\mu, \eta)=\frac{f_{0}^{l}(\mu)}{2}+\sum_{n=1}^{\infty} f_{n}^{l}(\mu) \cos n \eta$,

where

$$
\begin{aligned}
f_{n}^{l}= & B_{0}(-\alpha a)^{l} \frac{\sigma^{n}}{n !}\left\{\sum_{j=1}^{l}(\alpha a)^{-2 j} \sum_{i=0}^{\infty} u_{i}^{l, n,-j} \sigma^{2 i}\right. \\
& \left.+\sum_{j=0}^{\infty}(\alpha a)^{2 j} \sum_{i=0}^{\infty} u_{i}^{l, n, j} \sigma^{2(i+j)}\right\}
\end{aligned}
$$

with

$\sigma=e^{-\mu}$

$g_{l}$ and $u_{i}^{l, n, \pm j}$ are coefficients. The latter coefficients are fixed and they are given in Appendix A with some remarks on calculation procedures.

The coefficients $g_{l}$ are determined by the boundary condition, i.e. for us $B_{\mu}^{(\mathrm{T})}=0$ at $\mu=\mu_{0}$. The component $B_{\mu}^{(\mathrm{T})}$ from Eq. (25) explicitly is

$B_{\mu}^{(\mathrm{T})}=-\frac{1}{\alpha h_{\eta} h_{\varphi}} \sum_{n=1}^{\infty} n \sin n \eta \sum_{l=0}^{\infty} g_{l} f_{n}^{l}$.

The coefficient $g_{0}$ is arbitrary (scales the field) and can be set equal to 1 . The functions $f_{n}^{l}(n \geq 0)$ are constants at the boundary and we denote $\bar{f}_{n}^{l}=f_{n}^{l}\left(\mu_{0}\right)$. Writing Eq. (32) for the boundary and realizing that it must be fulfilled for every $\eta$, we get

$\sum_{l=1}^{\infty} g_{l} \bar{f}_{n}^{l}=-g_{0} \bar{f}_{n}^{0} \quad(n \geq 1)$.

This is a set of equations for $g_{l}$.

In previous cases, $\alpha$ was simply related to the flux rope size by Eq. (5). It resulted from the second requirement, axial field to 


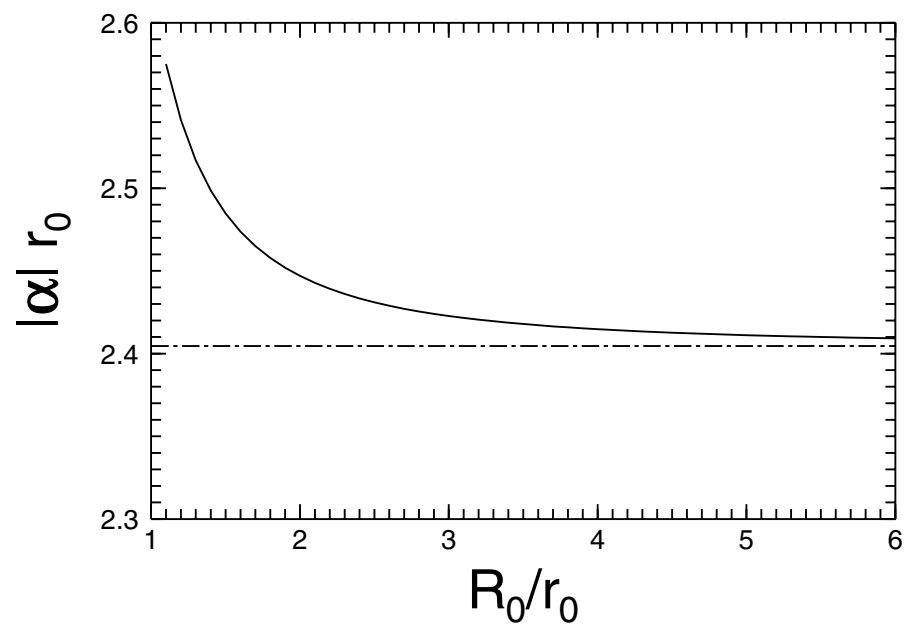

Fig. 2. Dependence of the force-free constant $\alpha$ on the aspect ratio $R_{0} / r_{0}$ of a toroid. For large aspect ratios, it tends to an asymptotic value, shown with the dashed-dotted line, which is consistent with the Lundquist solution. It is the first root of the Bessel function $J_{0}, a_{0}$.

Table 1. Alpha as a function of aspect ratio.

\begin{tabular}{ll|ll}
\hline \hline$R_{0} / r_{0}$ & $|\alpha| r_{0}$ & $R_{0} / r_{0}$ & $|\alpha| r_{0}$ \\
\hline 1.1 & 2.575 & 2 & 2.447 \\
1.2 & 2.541 & 2.5 & 2.431 \\
1.3 & 2.517 & 3 & 2.423 \\
1.4 & 2.499 & 4 & 2.415 \\
1.6 & 2.474 & 6 & 2.409 \\
1.8 & 2.458 & $\infty$ & 2.405 \\
\hline
\end{tabular}

be zero at the boundary, here $B_{\varphi}^{(\mathrm{T})}=0$ at $\mu=\mu_{0}$. Using Eq. (27) and utilizing Eq. (33), we get

$g_{0} \bar{f}_{0}^{0}+\sum_{l=1}^{\infty} g_{l} \bar{f}_{0}^{l}=0$.

This is an implicit equation for $\alpha$.

In fact, Eqs. (33) and (34) are intertwined and must be solved simultaneously. In practice, the summation is cut at some $L$. Equation (34) is solved by a root finding method (we used bi-section method, Press et al. 2002). For a given $\alpha$, the formula (33) represents a set of linear equations for $g_{l}, l=1, \ldots, L$, to be solved by a numerical method (we used singular value decomposition method, Press et al. 2002). With $g_{l}$ evaluated, $\alpha$ is checked if it fulfills Eq. (34) to a specified accuracy. For large aspect ratios, $|\alpha| r_{0}$ should go to $a_{0}$. Figure 2 shows how $|\alpha| r_{0}$ depends on the aspect ratio (cf. with Fig. 6 in Tsuji 1991). Some values are also tabulated in Table 1.

Magnetic helicity is defined for a magnetically closed body (which our toroid is) and it is given by

$H=\int \boldsymbol{A} \cdot \boldsymbol{B} \mathrm{d} V$,

where integration is over the body volume $V$ (in our case the toroid with $\mu=\mu_{0}$ ). For a linear force-free field, it simplifies into

$H=\frac{1}{\alpha} \int B^{2} \mathrm{~d} V$

where $B$ is the magnetic field magnitude. The helicity for the Tsuji solution (25)-(27) must be calculated numerically. For the other solutions presented here, it can be evaluated analytically.
Using Eq. (36), the calculation for the Miller \& Turner solution yields

$$
\begin{aligned}
H^{(\mathrm{MT})} & =\frac{2 \pi}{\alpha} \int_{0}^{2 \pi} \int_{0}^{r_{0}} B^{2} r\left(R_{0}+r \cos \theta\right) \mathrm{d} r \mathrm{~d} \theta \\
& =\frac{B_{0}^{2} \pi^{2} r_{0}^{3} R_{0}}{2 a_{0}}\left[8-\left(1+\frac{1}{a_{0}^{2}}\right) \frac{r_{0}^{2}}{R_{0}^{2}}\right] J_{1}^{2}\left(a_{0}\right) \operatorname{sign} \alpha,
\end{aligned}
$$

where sign is the signum function. Because the magnetic field is only approximately force-free, the helicity $H^{(\mathrm{MT})}$ given by Eq. (37) is also only approximate and valid for larger aspect ratios. The helicity per unit length is given by

$H_{l}^{(\mathrm{MT})}=\frac{H^{(\mathrm{MT})}}{2 \pi R_{0}}=\frac{B_{0}^{2} \pi r_{0}^{3}}{4 a_{0}}\left[8-\left(1+\frac{1}{a_{0}^{2}}\right) \frac{r_{0}^{2}}{R_{0}^{2}}\right] J_{1}^{2}\left(a_{0}\right) \operatorname{sign} \alpha$,

which reduces for large aspect ratios into

$H_{l}^{(\mathrm{MT})} \approx \frac{2 B_{0}^{2} \pi r_{0}^{3}}{a_{0}} J_{1}^{2}\left(a_{0}\right) \operatorname{sign} \alpha$.

The magnetic helicity for the modified Miller \& Turner solution is given by

$H^{(\mathrm{mMT})}=\frac{B_{0}^{2} \pi^{2} r_{0}^{3} R_{0}}{2 a_{0}}\left[8-\left(1-\frac{2}{a_{0}^{2}}\right) \frac{r_{0}^{2}}{R_{0}^{2}}\right] J_{1}^{2}\left(a_{0}\right) \operatorname{sign} \alpha$.

Equation (35) was used for the calculation because we know both the field (18) and its vector potential (17). The helicity (40) is exact. The helicity per unit length for large aspect ratios coincides with Eq. (39). The value in this equation also is the helicity per unit length for the toroidally adjusted Lundquist solution (that is, it does not depend on the aspect ratio in this case). A cylindrical flux rope is not a closed finite volume. Instead of helicity, the relative helicity per unit length is used for cylindrical flux ropes in literature $\left(H_{r} / L\right.$, e.g. Dasso et al. 2003). For the Lundquist field (2)-(4) the relative helicity per unit length coincides with the value given by Eq. (39).

\section{Comparison of toroidal solutions}

All solutions treated here are axisymmetric, so we shall compare fields at a cross section of a toroidal flux rope, which is circular with the radius $r_{0}$. The toroid is oriented that its rotational axis coincides with the $z$ axis and its circular axis inside the toroid lies in the $x y$ plane, where it forms a circle with the radius $R_{0}$ and its center is at the coordinate origin (Fig. 1). The Tsuji, Miller $\&$ Turner, and modified Miller \& Turner solutions are included into the comparison. The toroidally adjusted Lundquist solution, however, is not included. This solution does not depend on aspect ratio and its field distribution is circularly symmetric in the cross section, therefore it is simplified too much and qualitatively different from the other solutions.

Figures 3 and 4 show profiles of magnetic field magnitudes and components along two specific lines, one line is along the $x$ axis (Fig. 3), and the second line is parallel to the $z$ axis and crosses the $x$ axis in the distance $R_{0}$ from the origin (Fig. 4). One can see that the profiles are quite similar for all solutions. As can be expected, the largest deviations are for low aspect ratios and places near the toroid's hole; they are expressed more in the magnitude than in the components, and the modified Miller $\&$ Turner solution has a smaller difference from the Tsuji solution there. In addition to magnetic fields, the angle $\delta$ is plotted, 
M. Vandas and E. Romashets: Toroidal constant-alpha force-free fields
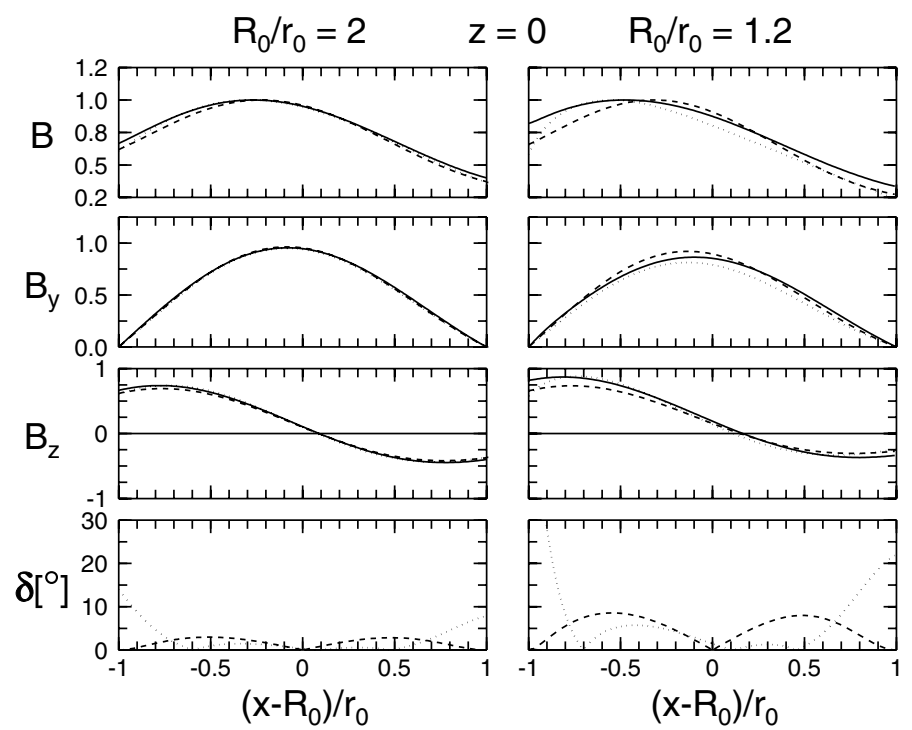

Fig. 3. Profiles of the magnetic field magnitude $B$, magnetic field components $B_{y}$ and $B_{z}$, and the $\delta$ angle along the $x$ axis for a toroidal flux rope with the Tsuji (solid lines), Miller \& Turner (dashed lines), and modified Miller \& Turner (dotted lines) solutions $(\alpha>0)$ ). The $B_{x}$ component is zero for all solutions, therefore, it is not shown. Magnetic field is scaled by maximum value inside the respective flux rope. The $\delta$ angle, the angle between $\boldsymbol{B}$ and $\operatorname{rot} \boldsymbol{B}$, indicates how the force-free condition is fulfilled; this angle is zero for the Tsuji solution. Two columns are plotted for two aspect ratios, 2 (left) and 1.2 (right).

which shows how the force-free condition is fulfilled (it is the angle between the field and current, which should be zero for an exactly force-free field). The modified Miller \& Turner solution fulfills this condition better in the inner parts of the flux rope than the Miller \& Turner solution, but there are large deviations at the outer parts where the Miller \& Turner solution is superior.

Figure 5 displays magnetic field magnitude distributions at circular cross sections of toroidal flux ropes for all three compared fields and two aspect ratios. In addition, in all cross sections there are six closed lines showing magnetic surfaces on which helical magnetic field lines lie. The first lines, the smallest ones and similar to small open circles, indicate positions of magnetic axes. The largest magnetic surfaces are circular and give the flux rope boundary. There are two symbols in each cross section. The "+" symbol indicates the geometric center of the cross section (the circle) and has coordinates $[0,0]$ in the plot. Here the toroid's circular axis crosses the $x z$ plane. The bullet determines the place of the maximum field magnitude $\left(B_{\max }\right)$. We see that positions of the magnetic axes are shifted to the right from the geometric center, away from the toroid's hole (cf. with Figs. 3 and 4 in Tsuji 1991), while positions of $B_{\max }$ are shifted to the left, toward the toroid's hole, and these shifts increase with decreasing aspect ratio. Their positions, and also the shapes of magnetic surfaces in the case of the modified Miller \& Turner solution, are more similar to the Tsuji solutions than it is in the case of the Miller \& Turner solution, even for low aspect ratios. However, there is a significant qualitative difference. Magnetic magnitude contours are prolate in the Tsuji field, while they are oblate in the Miller \& Turner field and its modification, and this difference is more pronounced in the former field.

Figure 6 is in the same format as Fig. 5, except the magnetic field magnitude is replaced by the $\delta$ angle and the Tsuji solution is missing because it has $\delta=0$ identically. The Miller \& Turner solution fulfills the force-free condition reasonably well, while the modified Miller \& Turner solution has large deviations

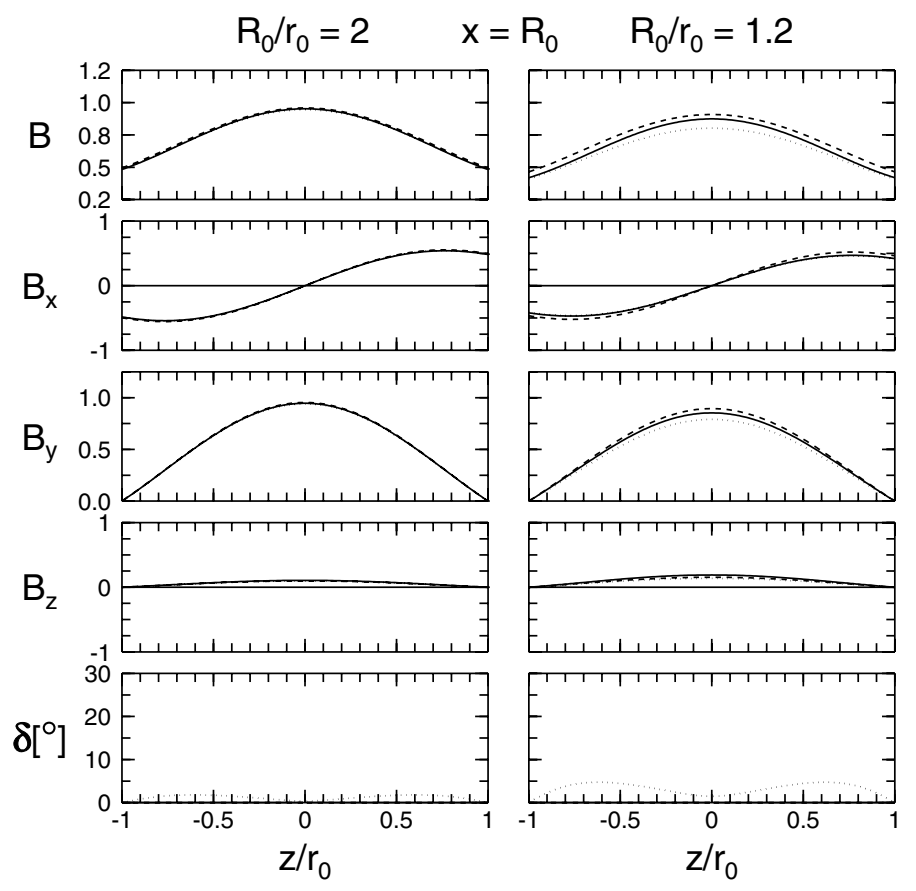

Fig. 4. Profiles of quantities along a line parallel to the $z$ axis and crossing the toroid's circular axis for a toroidal flux rope with the Tsuji (solid lines), Miller \& Turner (dashed lines), and modified Miller \& Turner (dotted lines) solutions $(\alpha>0)$. Format is similar to Fig. 3. The $\delta$ angle is zero for the Tsuji and Miller \& Turner solutions. Two columns are plotted for two aspect ratios, 2 (left) and 1.2 (right).

near the boundary, e.g. $\delta$ is over $60^{\circ}$ in a small region adjacent to the toroid's hole for the 1.2 aspect ratio case (near the point $[-1,0]$ in the figure). Figure 7 shows the $\delta$ angle averaged over the toroid's volume as a function of the aspect ratio for both solutions, which confirms the fact that the Miller \& Turner solution fulfills the force-free condition better.

Now we shall compare helicity of our toroidal flux ropes. In Fig. 8 a unitless quantity $h$ is plotted as a function of the aspect ratio for all three compared fields; $h$ is derived from the helicity as

$h=\frac{H}{\left(B_{\max }^{2} / \alpha\right) V}$,

where $V$ is the volume of the toroidal flux rope, and $V=$ $2 \pi^{2} R_{0} r_{0}^{2}, B_{\max }$ is the maximum field magnitude inside it. For example, for the Miller \& Turner solution it is

$h^{(\mathrm{MT})}=\left(\frac{B_{0}}{B_{\max }}\right)^{2}\left[2-\frac{1}{4}\left(1+\frac{1}{a_{0}^{2}}\right)\left(\frac{r_{0}}{R_{0}}\right)^{2}\right] J_{1}^{2}\left(a_{0}\right)$,

in the limit of large aspect ratios, we get

$h^{(\mathrm{MT})} \approx 2 J_{1}^{2}\left(a_{0}\right)$.

This quantity is also $h$ of the toroidally adjusted Lundquist solution and represents an asymptotic value of the other solutions for large aspect ratios (see the dashed-dotted line in Fig. 8). Comparing curves for three fields, we see that the formula (37) for the helicity derived from the Miller \& Turner solution approximates the correct dependence resulting from the Tsuji solution quite well, even for very low aspect ratios where the modified Miller \& Turner solution fails. 

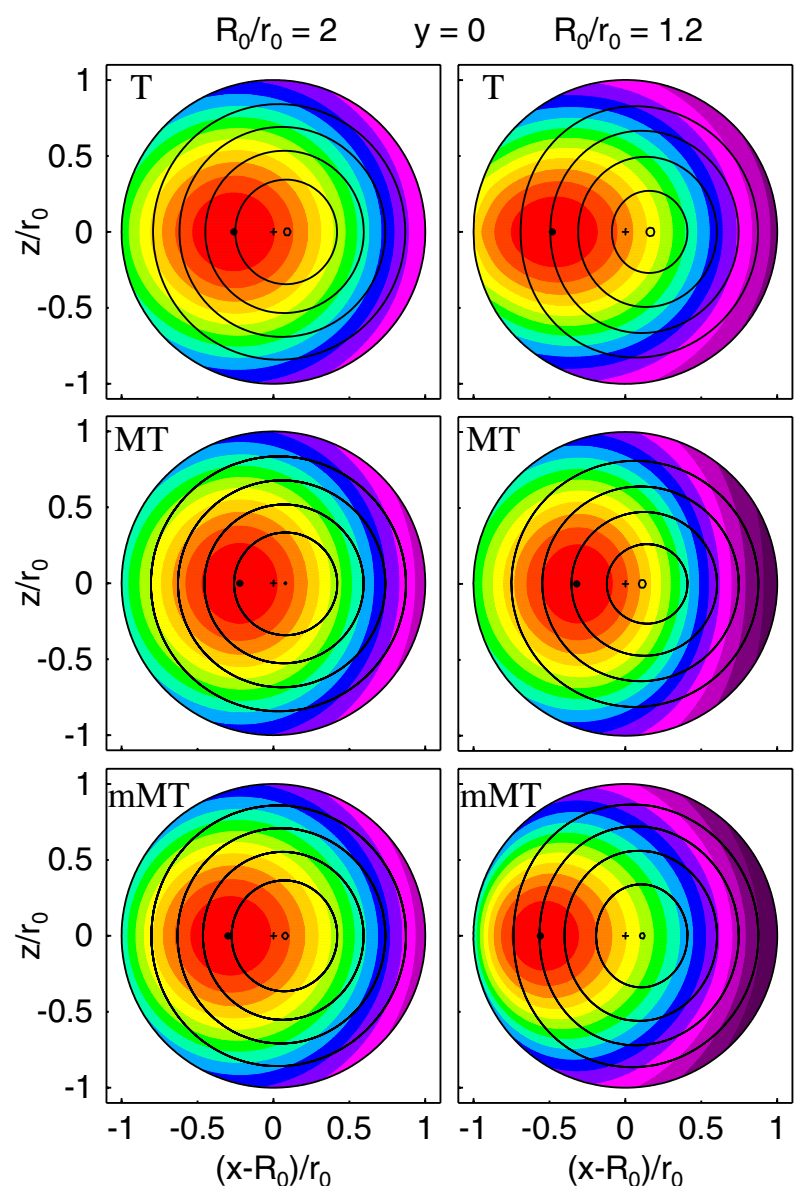

Fig. 5. Magnetic field magnitude distribution inside toroidal flux ropes (their circular cross sections) with the Tsuji (T), Miller \& Turner (MT), and modified Miller \& Turner (mMT) solutions (magnitude is scaled by the maximum value $B_{\max }$ inside a corresponding rope; the position of $B_{\max }$ shows the bullet). The ovals are magnetic surfaces (more precisely, their $x z$ cross sections) and the symbols "+" indicate geometric centers of the ropes. Two columns are plotted for two aspect ratios, 2 (left) and 1.2 (right).

\section{Conclusions}

Approximate constant-alpha force-free solutions in an ideal toroid, namely the toroidally adjusted Lundquist solution (Marubashi 1997), Miller \& Turner (1981) solution, and modified Miller \& Turner solution (Romashets \& Vandas 2003b), were compared with the exact solution (Tsuji 1991). The modified Miller \& Turner solution is exactly solenoidal for all aspect ratios, while the remaining two are only approximately solenoidal. For large aspect ratios $(>10)$, all solutions practically coincide. Comparing the magnetic field distribution at the flux-rope cross section, the field maximum is shifted toward the toroid's hole and the shift increases with the aspect ratio decrease. For low aspect ratios $(<3)$, the position of $B_{\max }$ is better matched by the modified Miller \& Turner than the Miller \& Turner field. The toroidally adjusted Lundquist field does not adequately describe the field distribution for smaller aspect ratios because it is always symmetric around the center of the cross section (with $B_{\max }$ at the center). The position of the magnetic axis is shifted in opposite direction and is satisfactorily matched by both the Miller \& Turner field and its modification. These two fields fulfill the force-free condition only approximately and, for small aspect ratios, the Miller \& Turner solution satisfies it much better. A simple formula for magnetic helicity was derived for
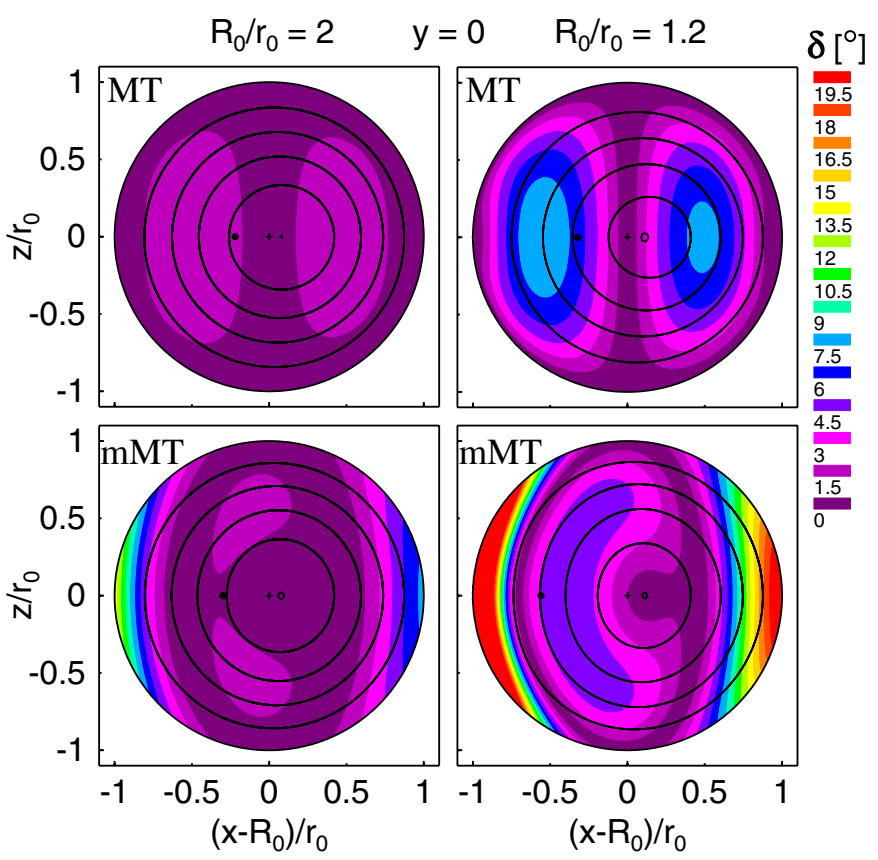

Fig. 6. Distribution of the $\delta$ angle inside toroidal flux ropes (their circular cross sections) with the Miller \& Turner (MT) and modified Miller \& Turner (mMT) solutions for two aspect ratios, 2 (left column), and 1.2 (right column).

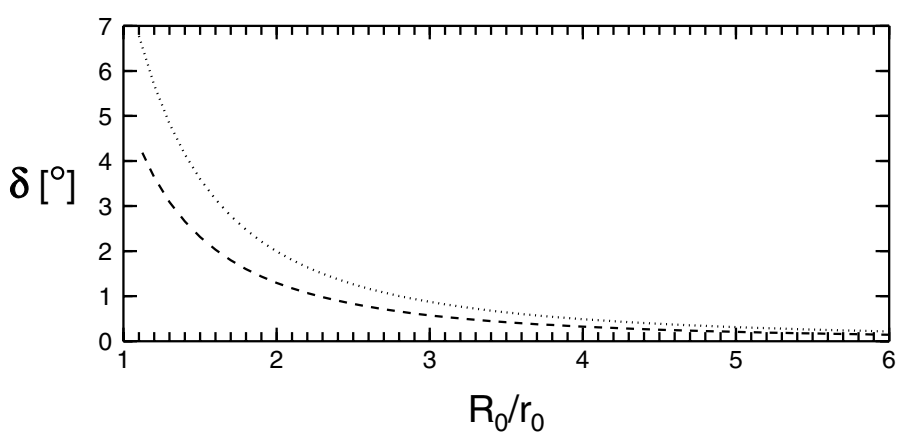

Fig. 7. The $\delta$ angle averaged over the toroid's volume for flux ropes with the Miller \& Turner (dashed line) and modified Miller \& Turner (dotted line) solutions as a function of aspect ratio $R_{0} / r_{0}$.

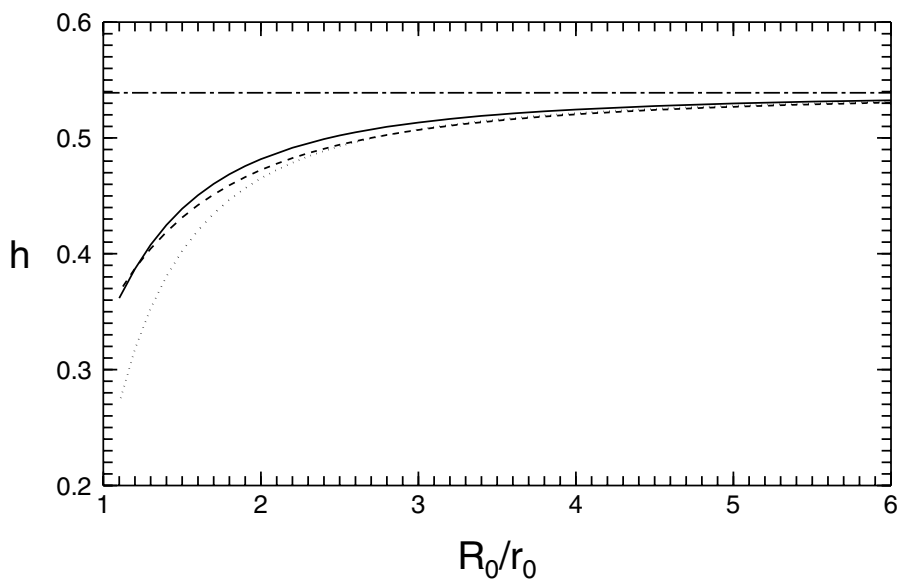

Fig. 8. Unitless quantity $h$ derived from the magnetic helicity (see text for the definition) as a function of the aspect ratio $R_{0} / r_{0}$ for toroidal flux ropes with the Tsuji (solid line), Miller \& Turner (dashed line), and modified Miller \& Turner (dotted line) solutions. The horizontal dashed-dotted line shows an asymptotic value to which all solutions tend for large aspect ratios. 
the Miller \& Turner field and its comparison with the accurate magnetic helicity calculated numerically from the Tsuji solution shows a reasonably good agreement even for very low $(<2)$ aspect ratios (contrary to the helicity derived from the modified Miller \& Turner solution, which significantly differs for them). One can conclude that the Miller \& Turner solution is a reasonable substitute for the exact solution even for low aspect ratios $(\approx 2)$. In magnetic cloud research, when there is a need to approximate magnetic cloud locally by a constant-alpha force-free toroid, the Miller \& Turner solution is a suitable magnetic field configuration for all aspect ratios met in practice.

Acknowledgements. This work was supported by project 14-19376S from GA ČR and by the AV ČR grant RVO:67985815.

\section{Appendix A: Coefficients of the Tsuji solution}

The coefficients $u_{i}^{l, n, j}$ are defined by

$$
\begin{aligned}
u_{i}^{l, n, j}= & \sum_{m=0}^{\min (i, l)} \sum_{k=m}^{\min (2 m, l)} \lambda_{m}^{l, k} d_{i-m}^{l-k, n, m+j}, \\
u_{i}^{l, n,-j}= & \sum_{m=0}^{j} \sum_{k=m}^{\min (l-j+m, 2 m)} \lambda_{m}^{l, k} d_{i}^{l-k, n, m-j} \\
& +\sum_{m=j+1}^{\min (j+i, l)} \sum_{k=m}^{\min (2 m, l)} \lambda_{m}^{l, k} d_{i-m+j}^{l-k, n, m-j}
\end{aligned}
$$

and valid for $j \geq 0$. The coefficients $d_{i}^{l, n, j}$ are given by

$d_{i}^{l, n, \pm j}=\frac{(-1)^{j} l !}{j !(l \pm j) !} \sum_{m=0}^{l} \frac{(-1)^{m}}{m !(l-m) !} c_{i}^{n, m, \pm j}$

where

$$
c_{i}^{n, m,-j}=\left(m-j+\frac{1}{2}\right)_{n}\left(m-j+\frac{3}{2}\right)_{2 j}
$$

$\times \sum_{k=0}^{i} \frac{\left(m-j+\frac{1}{2}\right)_{k}\left(n+m-j+\frac{1}{2}\right)_{k}\left(-m-\frac{1}{2}\right)_{i-k}\left(j-m-\frac{1}{2}\right)_{i-k}}{(n+1)_{k}(j+1)_{i-k} k !(i-k) !}$,

$c_{i}^{n, m, j}=\left(m+j+\frac{1}{2}\right)_{n}$

$\times \sum_{k=0}^{i} \frac{\left(m+j+\frac{1}{2}\right)_{k}\left(n+m+j+\frac{1}{2}\right)_{k}\left(-m-\frac{1}{2}\right)_{i-k}\left(j-m-\frac{1}{2}\right)_{i-k}}{(n+1)_{k}(j+1)_{i-k} k !(i-k) !}$.

The expression $(c)_{n}$ is the Pochhammer symbol or rising factorial, defined as $(c)_{n}=c(c+1) \ldots(c+n-1)$ with $(c)_{0}=1$. The coefficients $\lambda_{m}^{l, n}$ are defined for $l \geq 0,0 \leq m \leq l$, and $m \leq n \leq \min (2 m, l)$ by

$$
\begin{aligned}
\lambda_{0}^{0,0}= & 1 \\
\lambda_{m}^{l, n}= & -\frac{1}{d_{0}^{l-n, l-n, 0}}\left(\sum_{i=0}^{2 m-n-1} \sum_{k=\max (0, i+m-l)}^{i} \lambda_{i}^{l, 2 i-k} d_{0}^{l-2 i+k, l-n, i-m}\right. \\
& +\sum_{i=2 m-n}^{m} \sum_{k=\max (0, i+m-l)}^{2 m-n-1} \lambda_{i}^{l, 2 i-k} d_{0}^{l-2 i+k, l-n, i-m} \\
& \left.+\sum_{i=2 m-n}^{m-1} \lambda_{i}^{l, 2 i-2 m+n} d_{0}^{l-2 i+2 m-n, l-n, i-m}\right) \quad n \neq 2 m, l>0 \\
\lambda_{m}^{l, 2 m}= & \frac{l(l-2 m+1)_{m}}{(l-m) m !} l>0 .
\end{aligned}
$$

It is assumed in summations that when the upper limit is lower than the lower limit, the summation is not performed.

It holds for all $l \geq 0, i \geq 0, n \geq 0, j \geq 0$

$$
\begin{array}{rl}
d_{i}^{l, n, j}=0 & n+2 i<l, \\
d_{i}^{l, n,-j}=0 & n+2 i+2 j<l, \\
u_{i}^{l, n, j}=0 & n+i<l, \\
u_{i}^{l, n,-j}=0 & n+i+j<l .
\end{array}
$$

The coefficients $u_{i}^{l, n, j}$ do not depend on toroid's parameters; they can be pre-calculated. For numerical calculations, the unlimited sums are cut at some number, and we used 20. This number depends on value of aspect ratio that is to be reached: the lower aspect ratio is, the higher the number must be. Because the coefficients $\lambda_{m}^{l, n}$ are defined recursively, for given $l$, calculations are performed in increasing order of $m$ and then decreasing order of $n$. Here are some values to check implementation (all are exact numbers): $d_{0}^{2,3,-1}=-100.5, d_{2}^{6,4,0}=255.3, d_{1}^{7,3,-2}=-6252.75$, $\lambda_{2}^{4,3}=-32, \lambda_{3}^{4,4}=9, \lambda_{4}^{5,5}=7.5, \lambda_{2}^{6,4}=9, \lambda_{6}^{6,6}=9.84375$, $\lambda_{5}^{8,6}=2331, u_{0}^{1,1,-1}=0.75, u_{3}^{4,2,0}=0.4375, u_{2}^{7,3,-3}=97.453125$. Some additional values of $\lambda_{m}^{l, n}$ are listed in Table 1 of Tsuji (1991).

\section{References}

Burlaga, L. F. 1988, JGR, 93, 7217

Burlaga, L. F., \& Behannon, K. W. 1982, Sol. Phys., 81, 181

Burlaga, L., Sittler, E., Mariani, F., \& Schwenn, R. 1981, JGR, 86, 6673

Burlaga, L. F., Klein, L. W., Sheeley, Jr., N. R., et al. 1982, GRL, 9, 1317

Burlaga, L. F., Lepping, R. P., \& Jones, J. A. 1990, in Physics of Magnetic Flux Ropes, Geophys. Monogr. Ser., 58, eds. C. T. Russell, E. R. Priest, \& L. C. Lee (Washington, D.C.: AGU), 373

Cartwright, M. L., \& Moldwin, M. B. 2010, JGR, 115, A08102

Chen, J., \& Garren, D. A. 1993, GRL, 20, 2319

Dasso, S., Mandrini, C. H., Démoulin, P., \& Farrugia, C. J. 2003, JGR, 108, 8037 Hidalgo, M. A. 2013, ApJ, 766, 125

Hidalgo, M. A. 2014, ApJ, 784, 67

Ivanov, K. G., Harshiladze, A. F., Eroshenko, E. G., \& Styazhkin, V. A. 1989, Sol. Phys., 120, 407

Janvier, M., Demoulin, P., \& Dasso, S. 2013, A\&A, 556, A50

Klein, L. W., \& Burlaga, L. F. 1982, JGR, 87, 613

Krimigis, S., Sarris, E., \& Armstrong, T. 1976, Trans. AGU, 57, 304

Lepping, R. P., Jones, J. A., \& Burlaga, L. F. 1990, JGR, 95, 11957

Lepping, R. P., Berdichevsky, D. B., Wu, C.-C., et al. 2006, Ann. Geophys., 24, 215

Lepping, R. P., Wu, C.-C., \& Berdichevsky, D. B. 2015, Sol. Phys., 290, 553 Lundquist, S. 1950, Ark. Fys., 2, 361

Mandrini, C. H., Pohjolainen, S., Dasso, S., et al. 2005, A\&A, 434, 725

Marubashi, K. 1997, in Coronal Mass Ejections, Geophys. Monogr. Ser., 99, eds. N. Crooker, J. Joselyn, \& J. Feyman (Washington, D.C.: AGU), 147

Marubashi, K., \& Lepping, R. P. 2007, Ann. Geophys., 25, 2453

Miller, G., \& Turner, L. 1981, Phys. Fluids, 24, 363

Moldwin, M. B., Ford, S., Lepping, R., Slavin, J., \& Szabo, A. 2000, GRL, 27, 57

Press, V. H., Teukolsky, S. A., Vetterling, W. T., \& Flannery, B. P. 2002, Numerical Recipes in C, The Art of Scientific Computing, Second Edition (New York: Cambridge University Press)

Romashets, E. P., \& Vandas, M. 2003a, GRL, 30, 2065

Romashets, E. P., \& Vandas, M. 2003b, in Proc. ISCS 2003 Symp., Solar Variability as an Input to the Earth's Environment, ed. A. Wilson (Noordwijk: ESTEC), ESA SP, 535, 535

Shimazu, H., \& Marubashi, K. 2000, JGR, 105, 2365

Tsuji, Y. 1991, Phys. Fluids B, 3, 3379

Vandas, M., \& Romashets, E. P. 2010, in Twelve International Solar Wind Conf., eds. M. Maksimovic, K. Issautier, N. Meyer-Vernet, M. Moncuquet, \& F. Pantellini (Melville, New York: AIP), AIP Conf. Proc., 1216, 403

Vandas, M., Odstrcil, D., \& Watari, S. 2002, JGR, 107, 2156 